\title{
FlopR: An Open Source Software Package for Calibration and Normalization of Plate Reader and Flow Cytometry Data
}

\author{
Alex J. H. Fedorec, ${ }^{\S}$ Clare M. Robinson, ${ }^{\S}$ Ke Yan Wen, and Chris P. Barnes* \\ Cite This: https://dx.doi.org/10.1021/acssynbio.0c00296 \\ Read Online
}

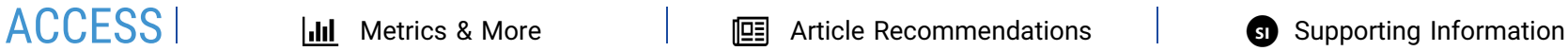

ABSTRACT: The measurement of gene expression using fluorescence markers has been a cornerstone of synthetic biology for the past two decades. However, the use of arbitrary units has limited the usefulness of these data for many quantitative purposes. Calibration of fluorescence measurements from flow cytometry and plate reader spectrophotometry has been implemented previously, but the tools are disjointed. Here we pull together, and in some cases improve, extant methods into a single software tool, written as a package in the $\mathrm{R}$ statistical framework. The workflow is validated using Escherichia coli engineered to express green fluorescent protein (GFP) from a set of commonly used constitutive promoters. We then demonstrate the package's power by identifying the time evolution of distinct subpopulations of bacteria from bulk plate reader data, a task previously reliant on laborious flow cytometry or colony counting experiments. Along with standardized parts and experimental methods, the development and dissemination of usable tools for quantitative measurement and data analysis will benefit the synthetic biology community by improving interoperability.

KEYWORDS: unit calibration, molecules of equivalent fluorophore, software tools, flow cytometry
$\mathrm{T}$ he construction of novel, reliable genetic circuits relies largely on the reproducible characterization of standard genetic parts. Fluorescence is often used as a quantitative output for engineering genetic circuits and can be measured in microplate readers or flow cytometers. Plate readers are particularly suited for high throughput applications, such as understanding temporal dynamics of multiple different constructs and conditions simultaneously. However, the data are bulk measurements that obscure population heterogeneity. Flow cytometry reveals this information but has more limited throughput capabilities. Recently, an inexpensive and easily implementable protocol to convert green fluorescence measurements from both instruments into intercomparable standard units of molecules of equivalent fluorescein (MEFL) per particle was validated across hundreds of laboratories in the international Genetically Engineered Machine (iGEM) competition. ${ }^{1}$ Measurements of standard calibrants are used to create calibration curves to convert the arbitrary units (a.u.) of individual machines to standardized units, a technique that has previously been established. ${ }^{2-4}$ Currently, however, software tools for calibration and normalization exist across multiple platforms and different coding languages including MATLAB (for example, TASBE Flow Analytics ${ }^{5}$ ), Python (CytoFlow ${ }^{6}$ and FlowCal ${ }^{7}$ ), R (flowBeads ${ }^{8}$ ), and Excel (iGEM's Plate Reader Calibration preformatted data sheet ${ }^{1}$ ). Table S1 compares the features between current free calibration software tools for plate reader and flow cytometry data.
Although this variety offers choice to end users, it makes highthroughput application difficult and time-consuming. In addition, autofluorescence normalization for plate reader measurements is often implemented inconsistently and in an "ad hoc" manner, even though it has been shown to be critical particularly when measuring fluorescent particles that share a similar emission wavelength with nonfluorescent cells. Consequently, standards for calibration and normalization have yet to be widely adopted and fluorescent data collected from microplate readers and flow cytometers is currently still often reported in arbitrary units. Our goal is to create a single, open source, easily usable tool to calibrate and normalize both plate reader and flow cytometer data to comparable standard units.

Here we present FlopR, a full calibration and normalization package in $\mathrm{R}$, the free and open source programming language, ${ }^{10}$ for both flow cytometry and microplate reader fluorescent data. FlopR normalizes raw data, then uses data from measurements of standard calibrants to generate

Received: June 3, 2020

Published: August 27, 2020 
Plate Reader Data Processing
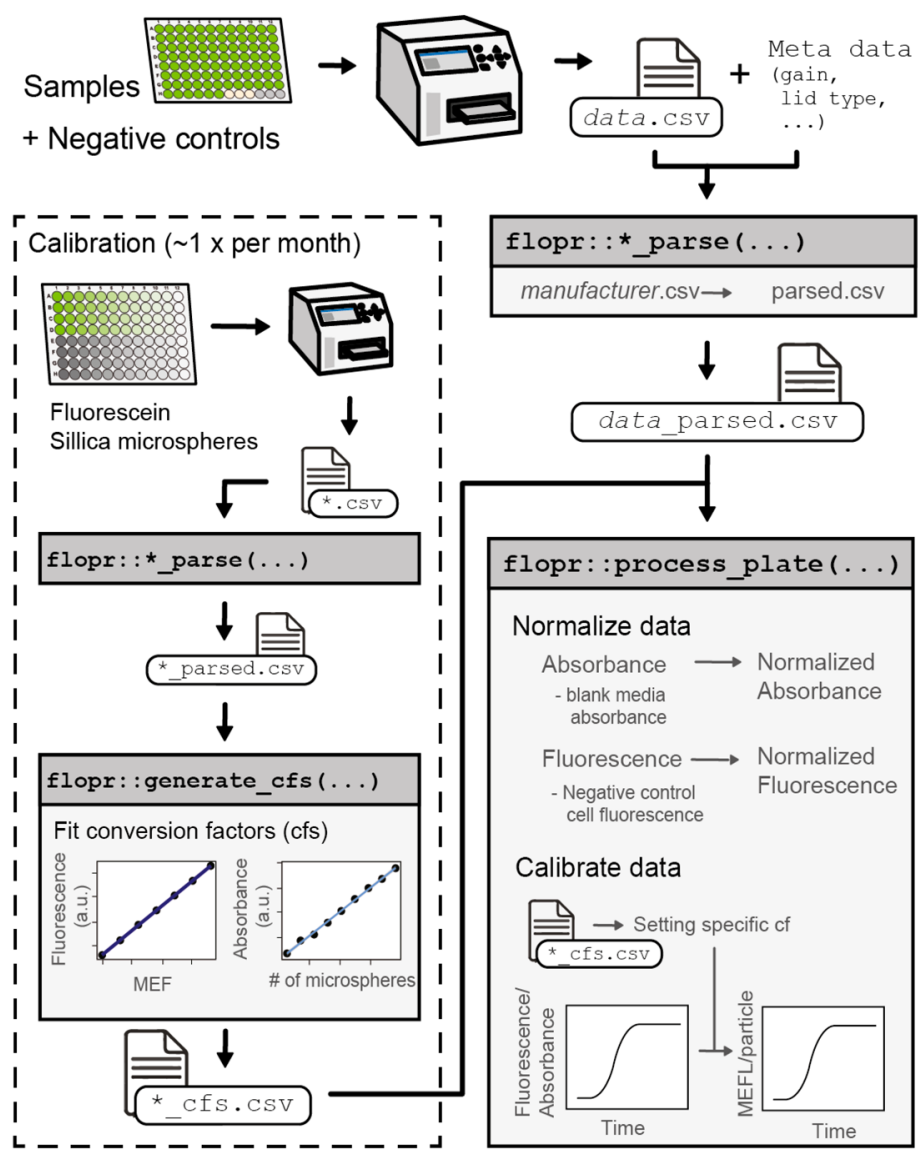

flopr: : _parse (...)

manufacturer.csv $\rightarrow$ parsed.csv
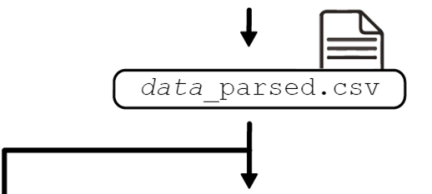

flopr::process_plate (...)

Normalize data

Absorbance $\longrightarrow$ Normalized

- blank media Absorbance

absorbance

Fluorescence $\longrightarrow$ Normalized

- Negative control Fluorescence

cell fluorescence

Calibrate data

$\rightarrow$ Setting specific cf

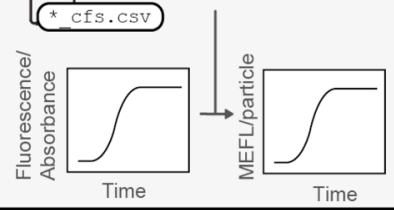

Flow Cytometry Data Processing
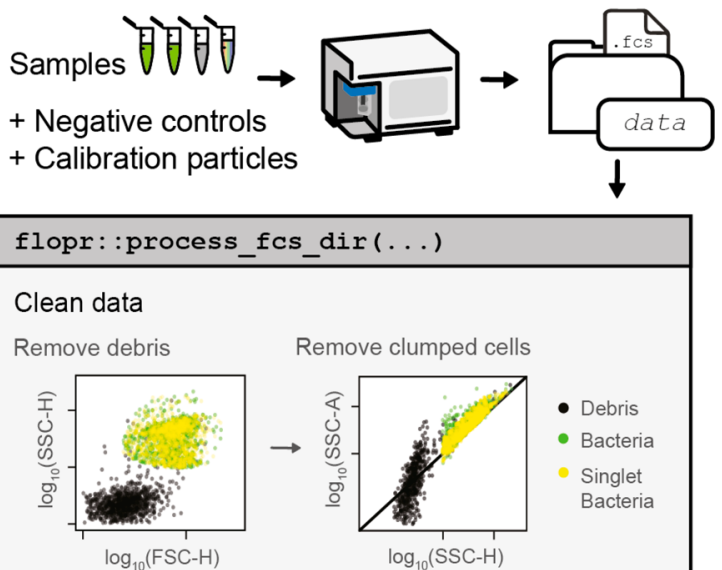

Normalize data
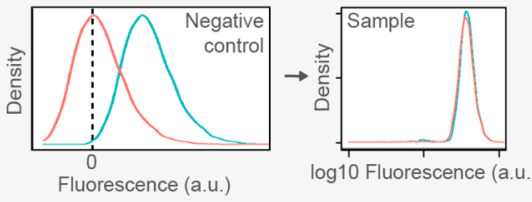

Raw data

Normalized

data

Calibrate data
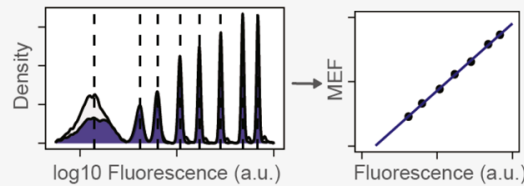

Fluorescence/ particle

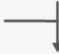

MEF/particle
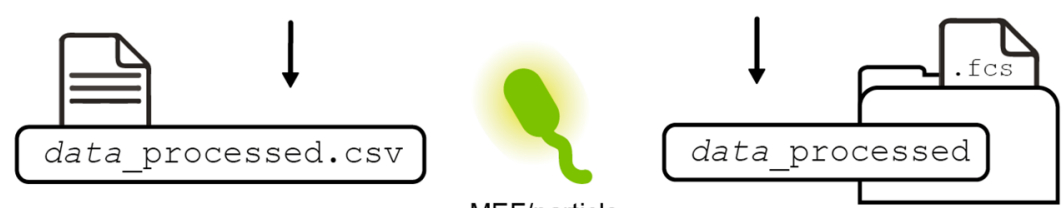

MEF/particle

Figure 1. Full plate reader and flow cytometry data processing by FlopR, including relevant function calls. FlopR normalizes and calibrates parsed plate reader sample data using the process_plate() function, and cleans, normalizes and calibrates .fcs flow cytometry data stored in a directory using the process_fcs_dir() function. FlopR outputs processed data in comparable, standard units of MEF/particle.

calibration curves and convert sample data recorded in arbitrary units to units of molecules of equivalent fluorophore (MEF) per particle. We demonstrate FlopR can accurately normalize and calibrate fluorescence data from $E$. coli that are constitutively expressing GFP to agree with normalized and calibrated literature values. Finally, we show FlopR can reconstruct the growth of individual fluorescent subpopulations of cells from plate reader data of heterogeneous bulk cell cultures, demonstrating its potential for complex and high throughput applications such as studies of dynamics within mixed microbial consortia.

\section{RESULTS}

Full Normalization and Calibration Workflow. FlopR has been designed to be simple to use for people with a limited background in programming. As such, it only requires one function call to normalize and calibrate an entire folder of flow cytometry data or a file containing microplate reader data. The full workflow is shown in Figure 1 and a detailed tutorial can be found on the GitHub page. As there is no common format for microplate reader data, a function to parse the data into a standard format is required. We provide an example parsing function, spark_parse(), which converts data from Tecan microplate readers.

Flow cytometry data, in the form of .fcs files, are processed using the process_fcs_dir() function. The function takes several arguments that control its behavior. A folder containing the .fcs files is specified and any file with a filename that matches the given pattern is processed. The data are first trimmed to remove debris and then singlet events are isolated from doublets and larger aggregates, as specified in the methods. If normalization and/or calibration is required, the names of the fluorescence channels to be processed are given. If a nonfluorescent control file is provided, it is first processed as above, after which the geometric mean of each fluorescence channel is calculated. These values are then negated from the other samples to provide normalized fluorescence values. If calibration of the flow cytometry data is desired, an .fcs file 


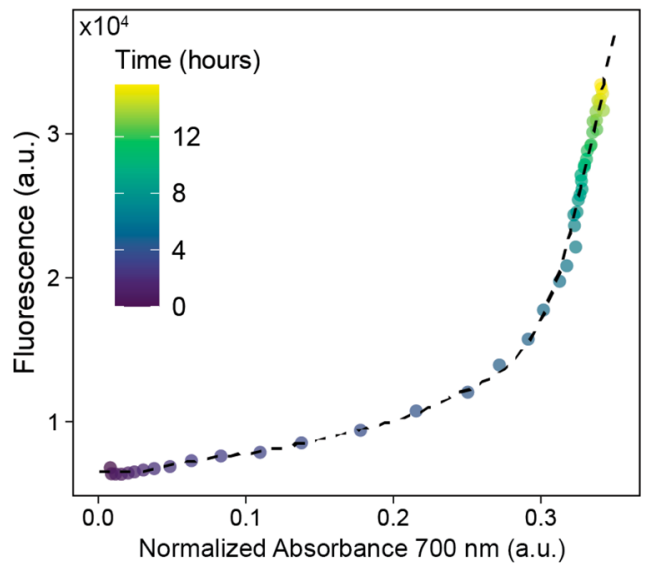

b

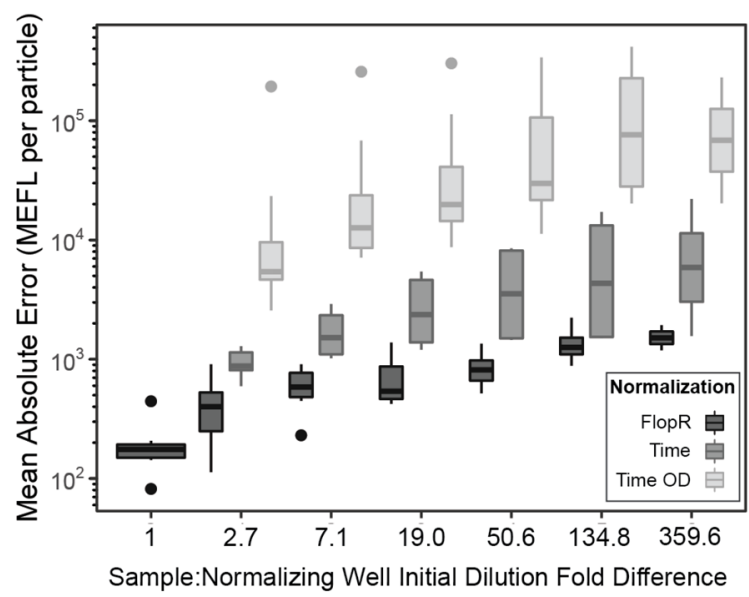

C
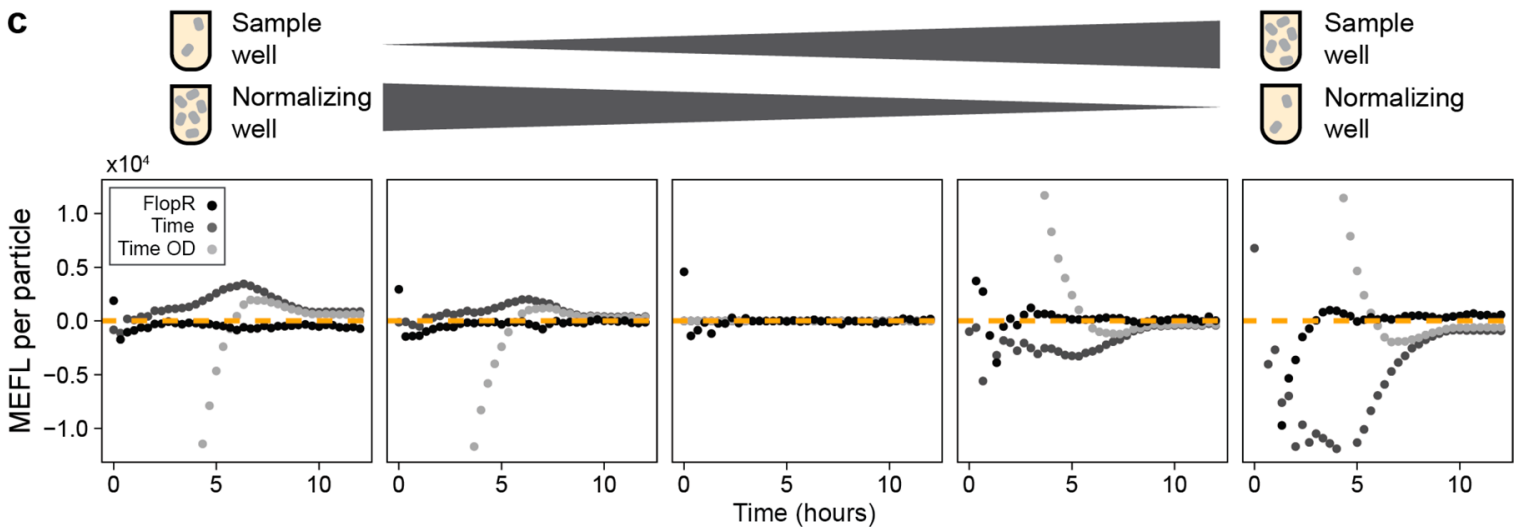

Figure 2. Fluorescence normalization in plate readers. (a) GAM fit of the absorbance $v$ s fluorescence of a well containing nonfluorescent control cells used for FlopR normalization. (b) Comparison of normalization performance by mean absolute error from the expected zero-fluorescence ground truth for nonfluorescent control cells using FlopR normalization, time normalization, or time OD normalization. Initial inoculates were serially diluted by a factor of 3:8. The fold difference is the pairwise initial dilution difference between sample and normalizing well. (c) MEFL per particle timecourses of nonfluorescent cells calculated using time normalization, time OD normalization, or FlopR normalization at different starting concentration of cells for the sample well and normalizing well. Orange dashed lines show the expected "ground truth" zero-fluorescence.

with "beads" as part of the filename must be in the folder. Calibration is performed using measurements of fluorescent beads labeled with known quantities of fluorophore. The peak values in each channel, produced by the bead manufacturer, need to be given to the function. If values are only given for a subset of the fluorescence channels, only those channels specified will be calibrated. A plot of the calibration curve for each fluorescent channel is produced along with the locations of the bead peaks identified by the software. If these peaks have not been correctly identified, the user can modify a function argument; increasing the value may be necessary if erroneous peaks have been identified. If the bead identification still fails, the bead peak locations can be specified manually. If desired, a plot will be saved showing the trimming, normalization, and calibration of the data. Each processed .fcs file will have additional columns for normalized and calibrated events, and is saved in a new folder.

The microplate reader workflow consists of two distinct parts: generation of calibration parameters, and generation and processing of sample data. The user should first prepare and measure a plate of standard calibrants (e.g., microspheres and fluorescein) at a range of dilutions, following the protocol developed for the iGEM competition ${ }^{1}$ and described in Supplementary Methods S2. The calibration data from the plate reader is first parsed and if using a Tecan plate reader the provided spark_parse() function can be used. The function needs to be provided with the path to the calibration data in the form of a .csv file, and the path to a .csv file detailing the layout of the calibration plate. This outputs a formatted .csv file. The generate_cfs () function uses the parsed calibration data to produce a .csv file that can then be used to calibrate later plate reader experiments. This protocol does not need to be carried out before every experiment but should be carried out regularly-roughly monthly-to ensure that the parameters reflect the machine's current behavior.

To process experimental plate reader data, the user should first create a .csv file with details of the samples and their position on the plate. The experimental data can then be parsed in the same way by providing the spark parse() function with the path to the data and layout .csv files. The parsed data can then be given to the process plate() function. One or more blank and nonfluorescent control wells can be specified. The names of the channels for absorbance and fluorescence measurements also need to be given. These should be the respective column names found in the input .csv file. We use a model based approach for autofluorescence normalization, detailed below, and allow the user to select the most appropriate model for their data. If the prior calibration protocol has been carried out, the experimental data can be converted to calibrated units. The gain level used for each fluorescence channel needs to be given along with the .csv file produced by generate_cfs( $)$. The output is saved as a .csv file 
containing all the original data, along with normalized and calibrated absorbance and fluorescence values.

Normalization of Autofluorescence in Plate Reader

Data. Careful normalization of plate reader data is required to correctly remove autofluorescence from the measurements of sample of interest. Incorrect normalization can diminish the dynamic range of the measured fluorescent output, particularly in the case of GFP. ${ }^{9}$ Often normalization is done simply by subtracting the fluorescence of a negative control consisting of nonfluorescent cells from the fluorescence of the cells of interest at the same time point. To calculate the fluorescence per OD, the normalized fluorescence is then divided by the blank-normalized OD. We describe this first, and most rudimentary, approach as "time normalization". This timebased normalization does not take into account growth differences between the nonfluorescent control and the sample population, which can be common in cells containing genetic circuits that may affect growth. ${ }^{11}$ A second normalization strategy, "Time OD normalization", takes the ratio of fluorescence to OD of both types of cells first, and then subtracts nonfluorescent cell measurements from those of the cells of interest. This can help account for differences in OD between the two types of cells but assumes a proportional relationship between OD and autofluorescence over time. Equations for "time normalization" and "time OD based normalization" are given in Supplemental Method S1. Another normalization approach involves using spectral unmixing of the main identified autofluorescent agents; ${ }^{9,12}$ however, this requires detailed knowledge of the source of the autofluorescence. ${ }^{9}$

Our approach is to fit an absorbance versus autofluorescence calibration curve of the nonfluorescent control cells (Figure $2 \mathrm{a})$, which gives autofluorescence as a function of absorbance over time for normalization. ${ }^{13-16}$ Previous mechanistic models have suggested that autofluorescence is proportional to the total volume of cells while flavin production and cell size remains constant. ${ }^{9}$ However, cell size is not constant across growth phases ${ }^{2,17}$ and production of flavins increases on approach to stationary phase. ${ }^{18}$ This means that autofluorescence is not linearly proportional to optical density. Indeed, we have observed that the relationship between OD and autofluorescence is monotonic from lag phase to early stationary phase, but during stationary phase autofluorescence increases while absorbance remains stable. Furthermore, over very long time periods, wells containing nonfluorescent cells can show a decrease in absorbance while maintaining the same level of autofluorescence (Figure S2). Due to the complexity of modeling this relationship, we chose a nonlinear smoothing approach to fit an autofluorescence calibration curve. The user can choose the best fitting nonlinear model, and has the options of a generalized additive model (GAM), ${ }^{19}$ locally estimated scatterplot smoothing (LOESS) model, $^{10}$ a secondorder polynomial or exponential model to fit the data. Extrapolation is a weakness of the two smoothing models. If the nonfluorescent control population does not grow as well as the samples, it may be better to choose one of the other models. However, this is an uncommon scenario in synthetic biology since engineered strains are often burdened by the circuits they carry.

In order to show quantitatively the performance of the different normalization approaches in cases of growth differences between normalized cells and normalizing cells, we grew nonfluorescent cells at different starting dilutions for $15 \mathrm{~h}$
(Figure S3). Accurate normalization of nonfluorescent cells should remove all autofluorescence, so the "ground-truth" fluorescence is expected to be zero (shown in Figure $2 \mathrm{c}$ as the orange dashed line). The mean absolute error (MAE) from the zero-fluorescence ground truth can then be used as an indicator of normalization performance. Pairs of wells, inoculated at different starting densities, were normalized against each other using the three different normalization methods (Figure S4), and their MAE calculated during the main growth phase of the sample well (Figure 2b). As the difference in starting dilution between the wells increases, the deviation from zero of both time normalization methods ("time normalization" and "time OD normalization") increases substantially. FlopR fitted normalization also shows a moderate increase but it is considerably smaller than the other types of normalization, indicating more consistent normalization performance, independent of growth. Figure $2 \mathrm{c}$ shows the normalized MEFL per particle of a subset of these nonfluorescent wells over time. In the middle panel, the sample well and normalizing well are identical indicating that all methods perform well. Time-based normalization methods are particularly prone to poor normalization at early time points, when delays or differences in the start of exponential growth of the two populations are emphasized.

Calibration of Plate Reader and Flow Cytometer Fluorescence Data. After normalization, FlopR converts the fluorescence of the cell sample of interest from arbitrary units to standardized units of MEF per particle by multiplying the normalized values with conversion factors generated from measurements of standard independent calibrants.

For the microplate reader calibration, measurements of a calibration plate of serial dilutions of fluorescein and microspheres measured prior to the main experiment are used as calibrants to generate conversion factors using FlopR's generate_cfs() function. Fluorescein shares similar excitation and emission wavelengths to green fluorescent protein (GFP), and the microspheres used are of approximately the same size and diameter as E. coli. ${ }^{2}$ Our protocol was adapted from the iGEM standard protocols ${ }^{20,21}$ and is available in the Supporting Information. The plate reader calibration plate is measured under all of the potential experimental conditions under which the fluorescent cells of interest will be measured in future experiments, including plate type, well volume or lid type, and with a range of gain settings. This is so that conversion factors generated from the calibration plate data will be valid for future experiments under a range of conditions. In particular, we note that the relationship between gain and conversion factors on the plate reader that we used was linear. This allows us to estimate the conversion factors if an experiment is run with a gain that was not calibrated against. To process data from the calibration plate, measurements are checked for validity to ensure they are not saturated and a model incorporating pipetting error ${ }^{1}$ is fitted to the remaining data. Plots are produced showing the fit for each measured condition, allowing the user to visually check the results.

For flow cytometry calibration, FlopR uses measurements of standard calibration particles (also called calibration beads). The calibration beads have known quantities of fluorophore bound to them and often present a range of intensities. To identify the bead fluorescence peaks measured by the flow cytometer, FlopR uses a Gaussian kernel density estimate from the R "stats" package, ${ }^{10}$ as opposed to Gaussian mixtures models or k-means clustering models used in other flow 
a

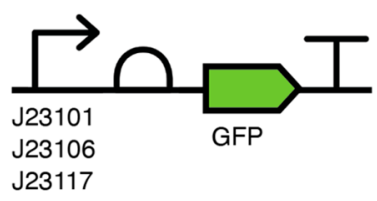

b

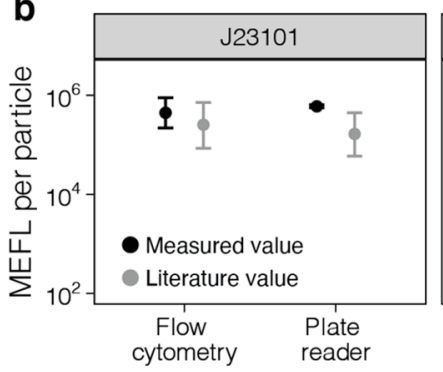

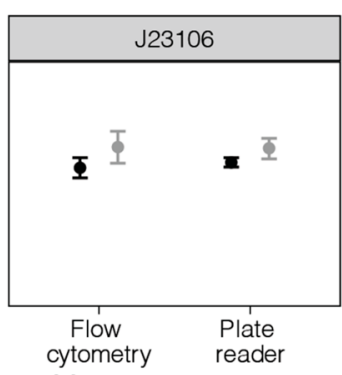

Measurement type

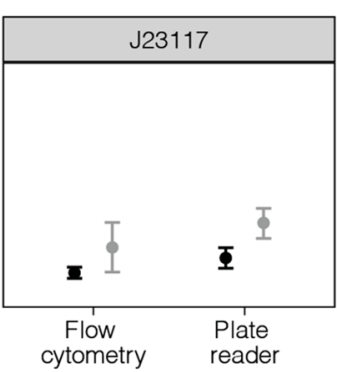

Figure 3. Demonstration of FlopR-produced MEFL per particle values measured by flow cytometry or on a plate reader. (a) Three different constitutive GFP expression constructs were tested, with promoters J23101, J23106, and J23117. (b) Fluorescence measurements on different days compared to literature values for the same plasmids. ${ }^{1}$ Plate reader data shows the geometric mean and standard deviation of 12 replicates on 2 days. Flow cytometry data shows the geometric mean fluorescence intensities and standard deviation of 11 replicates over 2 days.
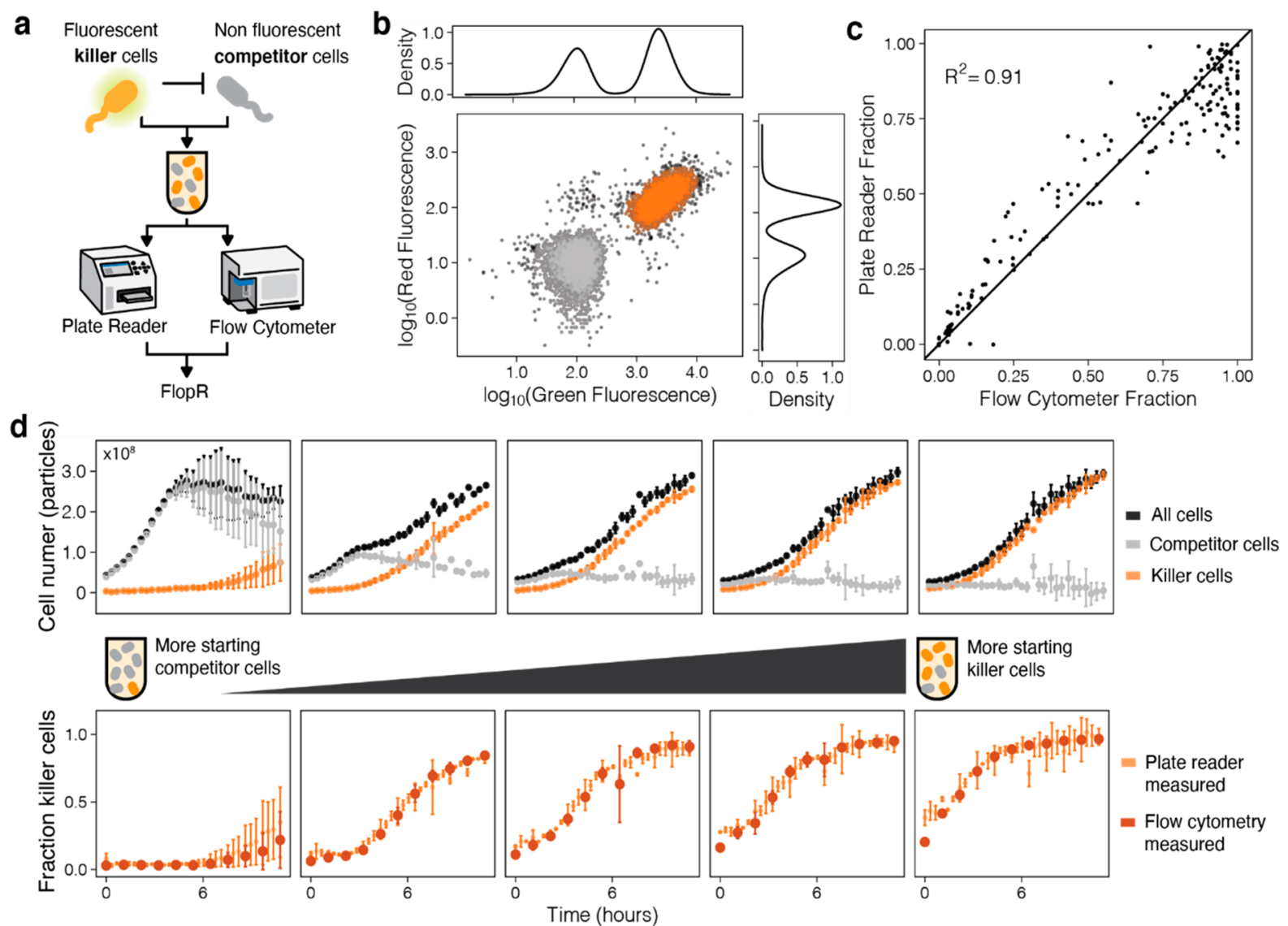

Figure 4. Using FlopR to monitor population dynamics of individual populations within heterogeneous bulk plate reader measurements. (a) Mixed cocultures of fluorescent killer cells and nonfluorescent competitor cells at different starting ratios were measured and compared using FlopR. (b) Distinct subpopulations are easily identifiable by clustering flow cytometry data. (c) Comparison of plate reader calculated killer cell fractions and flow cytometry measured killer cell population fractions for wells at identical time points. (d) Dynamics of individual cell populations (orange or gray for killer and competitor, respectively) reconstructed from plate reader data using FlopR. The top panel shows timecourses of cell number of strains reconstructed from plate reader data. The bottom panel shows timecourse data of killer cell population fractions calculated from plate reader data or measured from clustered flow cytometry data. Data for $(d)$ is mean and standard deviation of two replicate wells measured on the same day.

cytometry data calibration software. ${ }^{7,8}$ This allows us to provide the user with a single parameter to modify (the bandwidth, which is equal to the kernel standard deviation) if the default settings are unsuccessful at identifying bead peaks (Figure S1). The default bandwidth that we have specified (0.025) prevents over smoothing of narrow peaks at high fluorescence intensity, but details of when and how to change the bandwidth are in the tutorial. From experience with other software tools, even with the ability to control parameters, bead peak identification can fail, so we also provide the ability for users to manually specify peak locations. Corresponding values of MEF for each identified peak are provided by the calibration bead manufacturer and are input into FlopR by the user for the specific bead lot and brand used (full details available in the FlopR tutorial). The identified peaks are checked for validity by comparing the fold change in measured fluorescence of adjacent peaks to the expected fold change from the manufacturer and discarding those that are more than 
$25 \%$ outside what is expected. A model developed for FlowCal, ${ }^{7}$ which includes bead autofluorescence, is then fitted to produce coefficients for conversion between measured fluorescence in arbitrary units and calibrated fluorescence in MEF.

Demonstrating FlopR Function with Constitutive GFP Expression Data. We sought to demonstrate the use of FlopR using constructs that constitutively expressed GFP with differing strength promoters (Figure $3 a$ ). Figure $3 b$ shows the results of measurements of three different strength constructs on a plate reader and flow cytometer, compared against literature values of the same constructs. ${ }^{1}$ We followed the protocol conducted by the iGEM interlab study, in which only a single time point is measured, so it is worthwhile to note that these were normalized following the interlab protocol and were not normalized for autofluorescence according to FlopR's normalization fit. In this case the nonfluorescent control cells and GFP-expressing cells showed similar growth dynamics, so time-based normalization is expected to give accurate results. The results show that FlopR produces results that are not only consistent between replicates sampled on different days, but that are also comparable with results from the literature that were measured on different instruments and processed using other software.

Applying FlopR to Identify Bacterial Subpopulations in Bulk Plate Reader Measurements. Finally, we used FlopR's normalization capabilities to identify specific subpopulations of cells in mixed cultures to demonstrate its potential applications beyond green fluorescence calibration. There has been growing interest in engineering multicellular consortia; $;^{22,23}$ however, identifying the growth dynamics of individual strains within a mixed culture remains challenging. We use a previously described system ${ }^{24}$ in which bacteriocinproducing fluorescent "killer" cells were mixed at different starting fractions with nonfluorescent competitor cells, and use FlopR to process the data measured in a microplate reader and flow cytometer over $12 \mathrm{~h}$ (Figure 4a). Traditionally, separation of the two population fractions is only possible by clustering in flow cytometry data (Figure 4b), but sampling is manual and low throughput, and it requires different fluorescence proteins in either strain or high expression of a fluorescent protein in order to adequately distinguish between subpopulations. In this case, population fractions are simply calculated using the numbers of cells of each population in each cluster. The killer cells were engineered to produce two fluorescent proteins, GFP and mCherry, so that clustering could be performed using both fluorescence channels without the issue of spectral overlap of the competitor and killer cell populations when fluorescence expression is weak.

To reconstruct population fractions from plate reader data, we make the assumption that fluorescent cells in a monoculture are representative of the same cells in a coculture. With this assumption, we can use a positive control well composed of only fluorescent killer cells to create an absorbance-fluorescence calibration curve specific to killer cells using one of the fluorescence channels (Figure S5). This calibration curve allows us to take into account the fact that fluorescence per cell may not remain constant over time: a similar premise to the autofluorescence calibration curve described previously. At each time point, the fraction of killer cells was calculated by

$$
\text { fraction }(t)=\frac{n F_{\text {cells }}(t)}{n F_{\text {pos }}\left(n \mathrm{OD}_{\text {cells }}(t)\right)}
$$

where $n F_{\text {cells }}(t)$ is the normalized fluorescence of the sample at time $t, n \mathrm{OD}_{\text {cells }}(t)$ is the normalized absorbance of the sample at time $t$, and $n F_{\text {pos }}$ uses the calibration curve to get the expected fluorescence at the given absorbance assuming an entirely killer population. The calculated fraction of killer cells from measurements in both instruments are shown in the bottom panel of Figure 4d, and a comparison is presented in Figure $4 c$, both showing that FlopR can closely reconstruct accurate subpopulations of cells from bulk data. This fraction can then be multiplied by the calibrated particle count of the mixed wells to get the growth timecourses of individual subpopulations (top row of Figure 4d). At high starting fractions of killer cells, the competitor cells are immediately overwhelmed and the killer dominates the culture (right column of Figure 4d). However, at low initial killer cell fractions, the competitor cells experience an initial growth phase, until the concentration of bacteriocin is sufficiently large to begin eliminating the competitors. Reconstruction of cell population fractions from plate reader measurements does not require spectral separation of the two subpopulations and therefore the use of both fluorescent protein channels is not necessary. It is indeed possible to reconstruct cell fractions using the green fluorescence channel (Figure S6) giving similar results to the reconstruction using red fluorescence in Figure 4. The same coculture system was explored over a larger range of population ratios as well as a variety of starting population densities and the final plate reader calculated population fraction was compared with flow cytometry data (Figure S7). These results further demonstrate the accuracy of our method. However, the data also show that at low population densities the subpopulation fraction calculation may produce inaccurate estimates due to noisy measurement of absorbance in the plate reader at those densities.

We performed a sensitivity analysis to determine how the true population fractions, fluorescence intensities and absorbance values, along with errors in measurement of absorbance and fluorescence, affect our ability to correctly estimate population fractions (Supplementary Method S3). We approximate measurement error by assuming measurements are normally distributed around the true value and examine two scenarios: the error is proportional to the measurement, and the error is constant regardless of the magnitude of the measurement (Figure S8). In the former scenario a measurement error of $1 \%$ produces a sensitivity of 1 (all population estimates are within 5\% of the true value), a $2 \%$ error gives 0.94 and a $5 \%$ error gives 0.63 sensitivity. These hold regardless of the population ratios, fluorescence intensity or absorbance. However, when the measurement error is constant, the sensitivity decreases as the fluorescence intensity or absorbance decrease because the error proportionally gets larger. It is likely that true measurement error for plate readers is proportional to the magnitude of the measurement down to a minimum error. This would correspond with our observations in Figure S7 of accuracy diminishing at the lowest population densities.

\section{DISCUSSION}

FlopR is a free and open source software tool that allows direct comparison of flow cytometry and plate reader green fluorescence data. Future extensions to the software should 
include fluorescence calibration for colors other than green fluorescence. For example, calibrants for red fluorescence, such as TexasRed, are currently being investigated. ${ }^{25}$ Size calibration $^{26}$ - currently available in other flow cytometry packages ${ }^{5}$ - can also be added, which may be of particular use in order to understand changes or differences in morphology of different bacterial strains. Size calibration may be particularly important to understand the size of microsphere to use as a calibrant to avoid over- or under-estimation of population size, as differences in particle size can cause large differences in light scattering, particularly when the size of the particle approaches that of the incident light wave, as is the case with bacteria. ${ }^{2}$ Alternatively, the cells themselves could be used to create the calibration curves for specific strains: by measuring the number of cells in a known dilution in the flow cytometer, it would be possible to estimate the number of cells in a dilution series of the same sample measured in the plate reader. In addition, determination of the MEFL per individual GFP protein, for example by flow cytometry and liquid chromatography-mass spectrometry, ${ }^{27}$ would be useful to gain quantitative information on how many proteins per cells are producing that fluorescence. This would be highly beneficial to enable the coupling of computational models to experimental data. As this software is open source, it can also be extended with methods for calibration of other instruments and approaches, such as fluorescence microscopy, ${ }^{28}$ or other imaging techniques.

Measurement standards help reproducibility, transparency and collaboration within the scientific process. Despite this, fluorescence remains identified as a problematic measurement area. $^{29,30}$ We hope this tool will be of use to the synthetic biology community to facilitate and encourage the use of standardized units in fluorescence measurements. The full details on how to use the package and requirements can be found in the tutorial on FlopR's GitHub repository at www. github.com/ucl-cssb/flopr.

\section{METHODS}

Instruments and Settings. All plate reader experiments were done on a Tecan SPARK Multimode Microplate Reader, using 96 well black, clear bottom plates (Corning). Specific plate reader settings for the individual experiments of each figure are outlined in Tables S1. All fluorescence measurements were made from the top of the plate, with $125 \mu \mathrm{L}$ cell cultures. Plate reader data for Figures 3 and 5 was collected throughout the timecourse. Plate reader data for Figure $4 \mathrm{~b}$ was collected at a single time point, following the protocol detailed in Beal et al. and available at protocols.io., 11

All flow cytometry experiments were carried out on an Attune NxT Flow Cytometer. Green fluorescence was measured on the BL1 channel (excitation laser: $488 \mathrm{~nm}$, emission filter: $530 / 30 \mathrm{~nm}$ ), and red fluorescence on the YL2 channel (excitation laser: $561 \mathrm{~nm}$, emission: 620/15 nm). Cell cultures were diluted $1 \mu \mathrm{L}$ in $200 \mu \mathrm{L}$ of filtered PBS, with 3 mixing cycles and minimum 3 wash rinses between samples.

As is noted in Table S2, data for Figures 2 and 4 were measured at excitation and emission wavelengths of 485/20 $\mathrm{nm}$ and 535/20 nm for green fluorescence. These differ by 3 $\mathrm{nm}$ for excitation and $5 \mathrm{~nm}$ for emission from the flow cytometer excitation and emission wavelengths of the BL1 green fluorescence channel in the flow cytometer. However, as both the plate reader's excitation and emission bandwidths are $20 \mathrm{~nm}$ and the flow cytometer bandpass filter is $30 \mathrm{~nm}$, the small difference in the settings is negligible, and the measurements from both instruments are still comparable.

Calibration Materials. The full protocol for generating serial dilutions of plate reader calibrants is detailed in Supporting Method S1. Briefly, fluorescein isothiocyanate (Sigma-Aldrich, CAS: 3326-32-7) dissolved in PBS and 0.89 $\mu \mathrm{m}$ monodisperse silica microspheres (Cospheric: SiO2MS-2.0 0.890um-1g) suspended in microbiology grade water were used to create serial dilutions in a 96 well black, clear bottom plate (Corning), with four replicate dilution series for each calibrant. Due to the quick settling time of the microspheres, their dilutions were remixed by pipetting up and down immediately before measurement on the plate reader. For flow cytometry calibration, one drop of Spherotech Rainbow calibration particles (Biolegend catalog number: 422903, these are identical to Spherotech catalog number: RCP-305A (8 peaks) Spherotech lot number: 073112 ) was mixed with $300 \mu \mathrm{L}$ of PBS and measured using the same settings as the relevant experiment.

Strains and Cultures Conditions. All plasmids, strains, and antibiotic working concentrations used are listed in Table S3. For Figure 2 and 4 cells were cultured in M9 minimal media supplemented with $0.4 \%$ glycerol and $0.2 \%$ casamino acids throughout the experiments. Cells for the experiment in Figure $3 b$ were cultured in LB media throughout the experiment, following the standard iGEM protocol. ${ }^{31}$ For Figure 2, $5 \mathrm{~mL}$ cultures were grown overnight in $14 \mathrm{~mL}$ roundbottom vented cap culture tubes at $37{ }^{\circ} \mathrm{C}$ and $200 \mathrm{rpm}$ in a SciQuip ZHWY-103D incubator, then diluted 1:1000 in fresh M9 media to a total of $5 \mathrm{~mL}$ in a new $14 \mathrm{~mL}$ tube, grown for 6 more hours at $200 \mathrm{rpm}$, diluted in fresh M9 media to a target OD $700 \mathrm{~nm}$ of 0.1 and total volume of $5 \mathrm{~mL}$, then diluted stepwise by a factor of $3 / 8$ (for initial starting dilution ratios of $1,0.375,0.141,0.053,0.020,0.007,0.003$, and 0.001) and final well volume of $125 \mu \mathrm{L}$ per well in a 96 well black, clear bottom plate. For Figure 4, $5 \mathrm{~mL}$ overnight cultures were diluted 1:100 in fresh $\mathrm{M} 9$ media to a total of $5 \mathrm{~mL}$ in $14 \mathrm{~mL}$ flasks, then mixed at the specified ratios to a final volume of $125 \mu \mathrm{L}$ per well in a 96 well black, clear bottom plate.

Flow Cytometry Data "Trimming". Each file within the folder given as the dir_path argument is initially processed to remove debris using the flowClust package ${ }^{32}$ to discriminate between bacterial and debris populations using logged forwardscatter height versus side-scatter height. This uses a t-mixture model with a Box-Cox transformation to fit the clusters and integrated completed likelihood to determine if there is a debris population or not. If two populations are found, the one with the greater mean forward-scatter height is chosen as the sample population and the other is discarded as debris. If the data has already had debris gated out during the experimental setup, the user can indicate as such by setting the pre_cleaned argument to TRUE. In this case, the $10 \%$ outliers are removed, and the remaining events are chosen as the sample population. Next we attempt to remove doublets and larger cell aggregates using the singletGate() function provided by the flowStats package. ${ }^{33}$ This fits a linear model to logged side-scatter height versus side-scatter area and discards events outside the $90 \%$ confidence interval. The manipulation of the flow cytometry data within FlopR relies heavily on functions provided by the flowCore package. ${ }^{34}$

Flow Cytometry Normalization. The events from a nonfluorescent control are trimmed to remove debris and select singlets, as above. The geometric mean of each 
fluorescence channel is calculated, with $5 \%$ of observations from each end of the distribution removed prior in order to prevent skew from extreme values. ${ }^{5}$ Sample data are then normalized by negating these calculated values from each event in each fluorescence channel.

\section{ASSOCIATED CONTENT}

\section{SI Supporting Information}

The Supporting Information is available free of charge at https://pubs.acs.org/doi/10.1021/acssynbio.0c00296.

Normalization calculations; microsphere and fluorescein plate reader calibration protocol; subpopulation identification method; software comparison; plate reader settings; plasmids and strains; bead peak identification; raw absorbance data; pairwise normalizations; population fluorescence curves; populations using GFP; additional population data; sensitivity analysis (PDF)

\section{AUTHOR INFORMATION}

\section{Corresponding Author}

Chris P. Barnes - Department of Cell and Developmental Biology and UCL Genetics Institute, University College London, London WC1E 6BT, U.K.; (1) orcid.org/0000-0002-94591395; Email: christopher.barnes@ucl.ac.uk

\section{Authors}

Alex J. H. Fedorec - Department of Cell and Developmental Biology, University College London, London WC1E 6BT, U.K.; (1) orcid.org/0000-0003-0165-1705

Clare M. Robinson - Department of Cell and Developmental Biology, University College London, London WC1E 6BT, U.K.; () orcid.org/0000-0003-3581-2810

Ke Yan Wen - Department of Cell and Developmental Biology, University College London, London WC1E 6BT, U.K.; (1) orcid.org/0000-0001-7449-2624

Complete contact information is available at: https://pubs.acs.org/10.1021/acssynbio.0c00296

\section{Author Contributions}

AJHF and CPB conceived the idea. CMR, AJHF, and KYW performed all experiments. CMR and AJHF developed the software. CMR and AJHF performed analysis of the results. CMR and AJHF wrote the first draft of the manuscript. All authors contributed to manuscript revision, read, and approved the submitted version.

\section{Author Contributions}

${ }^{\S} \mathrm{AJHF}$ and CMR contributed equally.

\section{Notes}

All experimental data and plasmid sequences are available at 10.5281 /zenodo. 3977408 .

The authors declare no competing financial interest.

\section{ACKNOWLEDGMENTS}

The authors would like to thank Dr. Stefanie Frank (UCL) for providing plasmids from the iGEM biobrick registry plate distribution. $\mathrm{CPB}, \mathrm{AJHF}, \mathrm{KYW}$ received funding from the European Research Council (ERC) under the European Union's Horizon 2020 research and innovation program (Grant No. 770835). CPB, CMR received funding from the Wellcome Trust (209409/Z/17/Z).

\section{REFERENCES}

(1) Beal, J., Farny, N. G., Haddock-Angelli, T., Selvarajah, V., Baldwin, G. S., Buckley-Taylor, R., Gershater, M., Kiga, D., Marken, J., Sanchania, V., Sison, A., and Workman, C. T. (2019) Robust Estimation of Bacterial Cell Count from Optical Density. bioRxiv, Oct 13, 2019, 803239 (accessed Sep 1, 2020).

(2) Stevenson, K., McVey, A. F., Clark, I. B. N., Swain, P. S., and Pilizota, T. (2016) General Calibration of Microbial Growth in Microplate Readers. Sci. Rep. 6 (1), 38828.

(3) Vogt, Jr., R. F., Marti, G. E., and Zenger, V. (2008) Quantitative Fluorescence Calibration: A Tool for Assessing the Quality of Data Obtained by Fluorescence Measurements. In Standardization and Quality Assurance in Fluorescence Measurements I, pp 3-31, Springer, Berlin, Heidelberg. DOI: 10.1007/4243 2008_055.

(4) Beal, J., Haddock-Angelli, T., Baldwin, G., Gershater, M., Dwijayanti, A., Storch, M., de Mora, K., Lizarazo, M., and Rettberg, R. (2018) Quantification of Bacterial Fluorescence Using Independent Calibrants. PLoS One 13 (6), No. e0199432.

(5) Beal, J., Overney, C., Adler, A., Yaman, F., Tiberio, L., and Samineni, M. (2019) TASBE Flow Analytics: A Package for Calibrated Flow Cytometry Analysis. ACS Synth. Biol. 8 (7), 15241529.

(6) Teague, B. Cyto Flow, https://bpteague.github.io/cytoflow/ (accessed Jul 6, 2020)

(7) Castillo-Hair, S. M., Sexton, J. T., Landry, B. P., Olson, E. J., Igoshin, O. A., and Tabor, J. J. (2016) FlowCal: A User-Friendly, Open Source Software Tool for Automatically Converting Flow Cytometry Data from Arbitrary to Calibrated Units. ACS Synth. Biol. 5 (7), 774-780.

(8) Pontikos, N. (2020) FlowBeads: Analysis of Flow Bead Data, Bioconductor.

(9) Mihalcescu, I., Van-Melle Gateau, M., Chelli, B., Pinel, C., and Ravanat, J. L. (2015) Green Autofluorescence, a Double Edged Monitoring Tool for Bacterial Growth and Activity in Micro-Plates. Phys. Biol. 12 (6), 066016.

(10) R Core Team (2019) R: A Language and Environment for Statistical Computing, Vienna, Austria.

(11) Hoffmann, F., and Rinas, U. (2004) Stress Induced by Recombinant Protein Production in Escherichia Coli. Adv. Biochem. Eng./Biotechnol. 89, 73-92.

(12) Lichten, C. A., White, R., Clark, I. B. N., and Swain, P. S. (2014) Unmixing of Fluorescence Spectra to Resolve Quantitative Time-Series Measurements of Gene Expression in Plate Readers. BMC Biotechnol. 14, 11.

(13) Martin, Y., Page, M., Blanchet, C., and De Jong, H. (2019) WellInverter: A Web Application for the Analysis of Fluorescent Reporter Gene Data. BMC Bioinf. 20, 309.

(14) Stefan, D., Pinel, C., Pinhal, S., Cinquemani, E., Geiselmann, J., and de Jong, H. (2015) Inference of Quantitative Models of Bacterial Promoters from Time-Series Reporter Gene Data. PLoS Comput. Biol. 11 (1), No. e1004028.

(15) Berthoumieux, S., De Jong, H., Baptist, G., Pinel, C., Ranquet, C., Ropers, D., and Geiselmann, J. (2013) Shared Control of Gene Expression in Bacteria by Transcription Factors and Global Physiology of the Cell. Mol. Syst. Biol. 9, 634.

(16) Zaslaver, A., Bren, A., Ronen, M., Itzkovitz, S., Kikoin, I., Shavit, S., Liebermeister, W., Surette, M. G., and Alon, U. (2006) A Comprehensive Library of Fluorescent Transcriptional Reporters for Escherichia Coli. Nat. Methods 3 (8), 623-628.

(17) Volkmer, B., and Heinemann, M. (2011) Condition-Dependent Cell Volume and Concentration of Escherichia Coli to Facilitate Data Conversion for Systems Biology Modeling. PLoS One 6 (7), No. e23126.

(18) Surre, J., Saint-Ruf, C., Collin, V., Orenga, S., Ramjeet, M., and Matic, I. (2018) Strong Increase in the Autofluorescence of Cells Signals Struggle for Survival. Sci. Rep. 8, 12088.

(19) Wood, S. N. (2017) Generalized Additive Models: An Introduction with $R$, 2nd ed., Chapman and Hall/CRC. 
(20) Baldwin, G. S., Haddock-Angelli, T., Beal, J., Dwijayanti, A., Storch, M., Farny, N., Telmer, C., Vignoni, A., Tennant, R., and Rutten, P. Calibration Protocol - Plate Reader Fluorescence Calibration V.3 https://www.protocols.io/view/calibration-protocolplate-reader-fluorescence-cal-6zrhf56 (accessed Jul 8, 2020). DOI: $10.17504 /$ protocols.io.6zrhf56.

(21) Beal, J., Haddock-Angelli, T., Gershater, M., Sanchania, V., Buckley-Taylor, R., Baldwin, G. S., Farny, N., Tennant, R., and Rutten, P. Calibration Protocol - Plate Reader Abs600 (OD) Calibration with Microsphere Particles V.2 https://www.protocols. io/view/calibration-protocol-plate-reader-abs600-od-calibr549g8z6?version_warning=no (accessed Jul 8, 2020). DOI: $10.17504 /$ protocols.io.549g8z6.

(22) Johns, N. I., Blazejewski, T., Gomes, A. L. C., and Wang, H. H. (2016) Principles for Designing Synthetic Microbial Communities. Curr. Opin. Microbiol. 31, 146-153.

(23) Karkaria, B. D., Treloar, N. J., Barnes, C. P., and Fedorec, A. J. H. (2020) From Microbial Communities to Distributed Computing Systems. Front. Bioeng. Biotechnol. 8, 834.

(24) Fedorec, A. J. H., Karkaria, B. D., Sulu, M., and Barnes, C. P. (2020) Single strain control of microbial consortia. bioRxiv, DOI: 10.1101/2019.12.23.887331 (accessed Sep 1, 2020).

(25) Rutten, P., Telmer, C., Baldwin, G., Mishler, D. M., HaddockAngelli, T., Beal, J., Dwijayanti, A., Storch, M., Farny, N., Vignoni, A., and Tennant, R. iGEM Calibration Protocol - Red Fluorescent Proteins in Plate Readers https://www.protocols.io/view/igemcalibration-protocol-red-fluorescent-proteins-bcdjis $4 n$. DOI: $10.17504 /$ protocols.io.bcdjis4n.

(26) Koch, A. L., Robertson, B. R., and Button, D. K. (1996) Deduction of the Cell Volume and Mass from Forward Scatter Intensity of Bacteria Analyzed by Flow Cytometry. J. Microbiol. Methods 27 (1), 49-61.

(27) Speer, S. L., Guseman, A. J., Patteson, J. B., Ehrmann, B. M., and Pielak, G. J. (2019) Controlling and Quantifying Protein Concentration in Escherichia Coli. Protein Sci. 28 (7), 1307-1311.

(28) Baskin, D. S., Widmayer, M. A., and Sharpe, M. A. (2010) Quantification and Calibration of Images in Fluorescence Microscopy. Anal. Biochem. 404 (2), 118-126.

(29) Beal, J., Haddock-Angelli, T., Farny, N., and Rettberg, R. (2018) Time to Get Serious about Measurement in Synthetic Biology. Trends Biotechnol. 36 (9), 869-871.

(30) Beal, J., Goñi-Moreno, A., Myers, C., Hecht, A., del Carmen de Vicente, M., Parco, M., Schmidt, M., Timmis, K., Baldwin, G., Friedrichs, S., Freemont, P. S., Kiga, D., Ordozgoiti, E., Rennig, M., Rios, L., Tanner, K., de Lorenzo, V., and Porcar, M. (2020) The Long Journey towards Standards for Engineering Biosystems. EMBO Rep. 21, 1-5.

(31) Tennant, R., and Rutten, P. Standard iGEM Cell Measurement Protocol https://www.protocols.io/view/standard-igem-cellmeasurement-protocol-zgmf3u6. DOI: 10.17504/protocols.io.zgmf3u6 (accessed Sep 1, 2020).

(32) Lo, K., Hahne, F., Brinkman, R. R., and Gottardo, R. (2009) FlowClust: A Bioconductor Package for Automated Gating of Flow Cytometry Data. BMC Bioinf. 10, 145.

(33) Hahne, F., Gopalakrishnan, N., Khodabakhshi, A., Wong, C., and Lee, K. (2020) FlowStats: Statistical Methods for the Analysis of Flow Cytometry Data, Bioconductor. DOI: 10.18129/B9.bioc.flowStats.

(34) Ellis, B., Haaland, P., Hahne, F., Le Meur, N., Gopalakrishnan, N., Spidlen, J., Jiang, M., and Finak, G. (2020) FlowCore: Basic Structures for Flow Cytometry Data. 